\title{
Some remarks on the visible points of a lattice
}

\author{
M. BAake ${ }^{1), 2)}$, U. Grimm ${ }^{2), 3)}$, And D. H. Warrington ${ }^{4)}$ \\ 1) Institut für Theoretische Physik, Universität Tübingen, \\ Auf der Morgenstelle 14, 72076 Tübingen, Germany \\ 2) Department of Mathematics, The University of Melbourne, \\ Parkville, VIC 3052, Australia \\ 3) Instituut voor Theoretische Fysica, Universiteit van Amsterdam, \\ Valckenierstraat 65, 1018 XE Amsterdam, The Netherlands \\ 4) School of Materials, The University of Sheffield, \\ Mappin Street, Sheffield S1 3JD, Great Britain
}

\begin{abstract}
We comment on the set of visible points of a lattice and its Fourier transform, thus continuing and generalizing previous work by Schroeder [1] and Mosseri [2]. A closed formula in terms of Dirichlet series is obtained for the Bragg part of the Fourier transform. We compare this calculation with the outcome of an optical Fourier transform of the visible points of the 2D square lattice.
\end{abstract}

Recently, Mosseri has given a nice and elegant description of the set of visible points of a lattice (i.e., those points except the origin that connect to the origin via a straight line without hitting any other lattice point in between) and some of its properties [2], continuing previous work by Schroeder [1, [3]. Both authors are most interested in the Fourier transform of this set. Unfortunately, the results of these two attempts are contradictory. The algebraic approach of [2] is essentially correct, but contains a couple of mistakes and unnecessary restrictions. In what follows, we add several remarks to straighten this out and then compare the $2 \mathrm{D}$ case with an optical experiment.

Let $\Lambda=\mathbb{Z} b_{1} \oplus \cdots \oplus \mathbb{Z} b_{n}$ be a lattice in $n \mathrm{D}$ space with linearly independent basis vectors $b_{1}, \ldots, b_{n}$. The set $F_{\Lambda}$ of visible points [4, 3, 2] can be characterized as

$$
F_{\Lambda}=\left\{m_{1} b_{1}+\cdots+m_{n} b_{n} \mid \operatorname{gcd}\left(m_{1}, \ldots, m_{n}\right)=1\right\}
$$

where $g c d$ denotes the greatest common divisor. This set does not include the origin with respect to which it is defined. We follow [2] for the notation as far as possible.

The set $F_{\Lambda}$ is non-periodic and is left invariant by the group of lattice automorphisms, $\operatorname{Aut}(\Lambda)$, which is isomorphic to $G l(n, \mathbb{Z})$, the group of integer $n \times n$ matrices with determinant \pm 1 . This is seen from $M \in A u t(\Lambda)$ transforming fundamental cells of the lattice to other fundamental cells and hence visible points to visible points [4, 6]. As a consequence, the set of visible points admits precisely the same point symmetry as the lattice $\Lambda$ itself, the transformations are not restricted to pure rotations. This can clearly be seen from Fig. 1 which shows the case of the square lattice. 
How frequent are visible points? If $p$ denotes the probability of a lattice point to be visible (defined through a volume limit which exists, compare chapter 3.8 of [7]), we also have $(\ell \in \mathbb{N}=\{1,2,3, \ldots\})$ the probabilities

$$
P\left(x \in \ell \cdot F_{\Lambda}\right)=\frac{p}{\ell^{n}},
$$

because $\ell \cdot F_{\Lambda}=\left\{m_{1} b_{1}+\cdots+m_{n} b_{n} \mid \operatorname{gcd}\left(m_{1}, \ldots, m_{n}\right)=\ell\right\}$. On the other hand, we have

$$
\Lambda=\{0\} \cup \bigcup_{\ell=1}^{\infty} \ell \cdot F_{\Lambda},
$$

which is the correct version of eq. (1) in [2]. Note that (3) is the union of pairwise disjoint sets

The single point 0 , however, does not matter in the calculation of the probabilities wherefore we get

$$
1=\sum_{\ell=1}^{\infty} P\left(x \in \ell \cdot F_{\Lambda}\right)=p \cdot \sum_{\ell=1}^{\infty} \frac{1}{\ell^{n}} .
$$

We can thus write $p$ by means of Riemann's $\zeta$-function as

$$
p=\frac{1}{\zeta(n)}=\prod_{p \in \mathcal{P}}\left(1-\frac{1}{p^{n}}\right)
$$

where $\mathcal{P}$ denotes the set of primes 4 , 5]. Obviously, $p=0$ for $n=1$ (precisely two points are visible here), and $p$ rapidly approaches 1 with increasing $n$, compare Fig. 2. For even $n \geq 2$, the $\zeta$-function is known to be transcendental [8, 9] (and thus irrational) through $\zeta(2 m)=\frac{(2 \pi)^{2 m}}{2(2 m) !}\left|B_{2 m}\right|$, where $B_{2 m}$ are the Bernoulli numbers, compare [8]. Also, $\zeta(3)$ is irrational [10], while this question is open for odd $n>3$. Note that the nonperiodicity of $F_{\Lambda}$ follows from the irrationality of $p$, but not vice versa.

A different approach uses the 3rd Möbius inversion formula [3, [4 where one starts from the characteristic function $h_{\Gamma}$ of a set $\Gamma$, which is one for points of the set and zero otherwise, and rewrites Eq. (3) as

$$
h_{\Lambda}=h_{\{0\}}+\sum_{\ell=1}^{\infty} h_{\ell \cdot F_{\Lambda}} .
$$

Convergence is no problem because, for any given point, the right hand side is a finite sum. Now, using the inversion formula for such series [4] one obtains

$$
h_{F_{\Lambda}}=\sum_{\ell=1}^{\infty} \mu(\ell) h_{\ell \cdot \Lambda \backslash\{0\}}
$$

with the Möbius function $\mu(\ell)$, cf. [四]. Note the differences to eqs. (1-3) in [2] which result from the little mistake in the description of the lattice $\Lambda$. It is important though because $\left|\sum_{\ell=1}^{N} \mu(\ell)\right|$ does not converge: it diverges faster than $N^{1 / 2}$ [3, 11].

\footnotetext{
${ }^{1}$ This property would be destroyed by the (unconventional) addition of the origin to the set $F_{\Lambda}$.
} 
This indicates that the calculation of the Fourier transform requires some care: it is not clear that the set of visible points is strictly almost periodic in the sense that the Fourier transform need not be a sum over $\delta$-distributions (or Bragg peaks) only. In fact, for $n=1$, this diffractive part vanishes due to the existence of only 2 visible points and the Fourier transform is $2 \cos (k x)$ and thus continuous. This limit must nevertheless be covered by the correct treatment. In [2], this is hidden by an infinite normalization factor - the formula would give vanishing Fourier transform for $n=1$.

Let us now consider the so-called structure factor in more detail. The structure factor $S_{\Gamma}$ of a discrete set $\Gamma$ is the Fourier transform of $\delta$-scatterers of equal strength on all points of $\Gamma$, i.e.,

$$
S_{\Gamma}(k)=\int \sum_{x \in \Gamma} \delta\left(x^{\prime}-x\right) e^{-i k x^{\prime}} d x^{\prime}=\sum_{x \in \Gamma} e^{-i k x} .
$$

If $\Gamma$ has inversion symmetry (as $\Lambda$ and $F_{\Lambda}$ do), we can also write $S_{\Gamma}(k)=\sum_{x \in \Gamma} e^{i k x}=$ $\sum_{x \in \Gamma} \cos (k x)$. Now, with Eq. (7), we find

$$
S_{F_{\Lambda}}(k)=\sum_{x \in \Lambda} \sum_{\ell=1}^{\infty} \mu(\ell) h_{\ell \cdot \Lambda \backslash\{0\}}(x) \cdot e^{i k x},
$$

to be understood in the distribution sense. Nevertheless, due to lack of convergence, an interchange of the sums is a subtle business. Though a complete treatment is desirable here, it is far beyond the scope of this comment and therefore left to a forthcoming publication [12]. But in the spirit of [2], one can indeed extract the point-like contributions, henceforth called Bragg peaks (though we are talking about amplitudes rather than intensities here) because other contributions are arbitrarily small in comparison to the $\delta$-peaks in the thermodynamic limit.

Before we continue, let us remark that the structure factor $S_{F_{\Lambda}}(k)$ is periodic in $k$ w.r.t. the reciprocal lattice $\Lambda^{*}$ : we can alternatively write

$$
S_{F_{\Lambda}}(k)=\int \sum_{x \in \Lambda} \delta\left(x^{\prime}-x\right) \cdot h_{F_{\Lambda}}\left(x^{\prime}\right) e^{-i k x^{\prime}} d x^{\prime}
$$

with the characteristic function $h_{F_{\Lambda}}$ defined above. But then, $S_{F_{\Lambda}}(k)$ is the convolution of $S_{\Lambda}(k)$ - which is periodic ! - with the Fourier transform of the characteristic function $h_{F_{\Lambda}}$. Consequently, $S_{F_{\Lambda}}(k)$ itself is periodic. Furthermore, since the set $F_{\Lambda}$ of visible points is invariant under $A u t(\Lambda)$, the corresponding property applies to its Fourier transform, which is thus invariant under $A u t\left(2 \pi \Lambda^{*}\right)$ - the latter again being isomorphic with the group $G l(n, \mathbb{Z})$. These two arguments apply to the full structure factor (and also to $n=1$ ), not only to its Bragg peaks.

To describe the latter, we now consider the reciprocal lattice $2 \pi \Lambda^{*}$ with $\Lambda^{*}=$ $\{y \mid x \cdot y \in \mathbb{Z} \quad \forall x \in \Lambda\}=\mathbb{Z} b_{1}^{*}+\cdots+\mathbb{Z} b_{n}^{*}, b_{i}^{*} \cdot b_{j}=\delta_{i j}$, a Bragg peak sits on every $k=2 \pi\left(r_{1} b_{1}^{*}+\cdots+r_{n} b_{n}^{*}\right)$ with rational coefficients, i.e., $r_{i} \in \mathbb{Q}$. If we write $r_{i}=p_{i} / q_{i}$ with coprime integers $p_{i}, q_{i}$ (where we take $q_{i}>0$ for convenience), the peaks have strength $H=H\left(q_{1}, \ldots, q_{n}\right)$,

$$
H\left(q_{1}, \ldots, q_{n}\right)=\sum_{m=1}^{\infty} \frac{\mu(m a)}{(m a)^{n}}, a=\operatorname{lcm}\left(q_{1}, \ldots, q_{n}\right),
$$


relative to the Bragg peaks of the lattice $\Lambda$. Here, $l \mathrm{~cm}$ denotes the least common multiple. In particular, $q_{1}=\ldots=q_{n}=1$ gives

$$
H(1, \ldots, 1)=\sum_{m=1}^{\infty} \frac{\mu(m)}{m^{n}}=\frac{1}{\zeta(n)},
$$

which is again the frequency of visible points, while $H$ is zero unless all arguments are square free, because the Möbius function vanishes for numbers that are divisible by a square. This explains the "dark" lines in the diagram of intensities (i.e., lines without spots - hence "white" in Fig. 3 while really dark in Fig. 4) which are given - in kinematic approximation - by the absolute squares of the amplitudes (11).

Eq. (11) gives a closed formula for the amplitudes of the Bragg peaks in form of an infinite sum, but can still be simplified considerably. If we consider

$$
f(n, a)=\sum_{\ell=1}^{\infty} \frac{\mu(\ell a)}{(\ell a)^{n}}
$$

for $\ell, a \in \mathbb{N}$, we can use standard techniques from the treatment of Dirichlet series, compare [5], to evaluate the sum. If $a=p_{1} \cdot \ldots \cdot p_{r}$ is the product of pairwise distinct primes, one can easily show by induction that

$$
f\left(n, p_{1} \cdot \ldots \cdot p_{r}\right)=\frac{f(n, 1)}{\prod_{j=1}^{r}\left(1-p_{j}^{n}\right)},
$$

and hence, with the multiplicativity of the Möbius function and Eq. (13),

$$
f(n, a)=\frac{\mu(a)}{a^{n}} \cdot \prod_{p \nmid a, p \in \mathcal{P}}\left(1-\frac{1}{p^{n}}\right)=\frac{1}{\zeta(n)} \cdot \frac{\mu(a)}{\prod_{p \mid a, p \in \mathcal{P}}\left(p^{n}-1\right)} .
$$

Inserting this into (11) finally gives, with $a=\operatorname{lcm}\left(q_{1}, \ldots, q_{n}\right)$, the amplitude function

$$
H\left(q_{1}, \ldots, q_{n}\right)=H(1, \ldots, 1) \cdot \frac{\mu(a)}{\prod_{p \mid a, p \in \mathcal{P}}\left(p^{n}-1\right)} .
$$

This way, the calculation of the amplitudes relative to the central amplitude is reduced to the evaluation of a finite product, compare Fig. 3 for the 2D example of the square lattice. There, a Bragg peak is represented by a dot with radius proportional to the amplitude (i.e., the area is proportional to the intensity).

The Fourier transform is periodic, although the set itself obviously is not - it is not even quasiperiodic because the Fourier module (i.e., the $\mathbb{Z}$-module generated by the positions of the $\delta$-peaks) is only countably but not finitely generated. The key structure is thus contained in one fundamental domain of the reciprocal lattice. This is indeed clearly seen in Figs. 3 and 4 . The latter was obtained by optical Fourier transform of a finite portion of $F_{\mathbb{Z}^{2}}$ on an optical bench. Here, we used some 9000 points of radius $15 \%$ of the lattice constant. They were prepared as a negative slide made from $24 \times 36 \mathrm{~mm}^{2}$ documentary film. The light was extracted from a laser beam, widened by a high quality lens to a parallel beam covering the entire slide. Although the image is not perfect (one should not expect rapid convergence 
in view of the remarks after (9)) and still shows some standard optical errors, it is nevertheless amazing how clear the structure with the subtle invariance properties is recovered. It is correctly described by the approach via the Möbius transform while previous numerical attempts [1] seem to fail for reasons we were not able to unravel.

Of course, one can now deal with generalizations like the set of points visible to two or more observers which resembles the above situation in many respects, although the relative position of the observers does play a significant role. Unfortunately, we could not find similarly nice closed expressions for the Fourier transform wherefore we drop further details here.

\section{Acknowledgements}

We thank P. A. B. Pleasants, M. Schlottmann and C. Sire for interesting discussions on the subject and J. Bachteler and D. Joseph for critically reading the manuscript. Financial support from Deutsche Forschungsgemeinschaft is gratefully acknowledged.

\section{References}

[1] M. R. Schroeder, A simple function and its Fourier transform, Mathem. Intelligencer 4 (1982) 158-61.

[2] R. Mosseri, Visible points in a lattice, J. Phys. A25 (1992) L25-9.

[3] M. R. Schroeder, Number Theory in Science and Communication, 2nd ed., 2nd printing, Springer, Berlin (1990).

[4] G. H. Hardy and E. M. Wright, An Introduction to the Theory of Numbers, 5th ed., Clarendon Press, Oxford (1979).

[5] E. C. Titchmarsh, The Theory of the Riemann Zeta-Function, reprint of the 2nd ed., Clarendon Press, Oxford (1967).

[6] C. L. Siegel, Lectures on the Geometry of Numbers, Springer, Berlin (1988).

[7] T. M. Apostol, Introduction to Analytic Number Theory, Springer, New York (1976).

[8] M. Abramowitz and I. A. Stegun, Handbook of Mathematical Functions, Dover, New York (1965).

[9] T. M. Apostol, A Proof that Euler Missed: Evaluating $\zeta(2)$ the Easy Way, Mathem. Intelligencer 5 (1983) 59-60, and references therein.

[10] R. Apéry, Irrationalité de $\zeta(2)$ et $\zeta(3)$, Soc. Mathem. France Astérisque 61 (1979) 11-3;

F. Beukers, A Note on the Irrationality of $\zeta(2)$ and $\zeta(3)$, Bull. London Math. Soc. 11 (1979) 268-72.

[11] A. M. Odlyzko and H. J. J. te Riele, Disproof of the Mertens conjecture, J. reine angew. Math. 357 (1985) 138-60.

[12] M. Baake, R. V. Moody and P. A. B. Pleasants, Diffraction from visible lattice points and kth power free integers, Discr. Comput. Geom. (1999), to appear. 

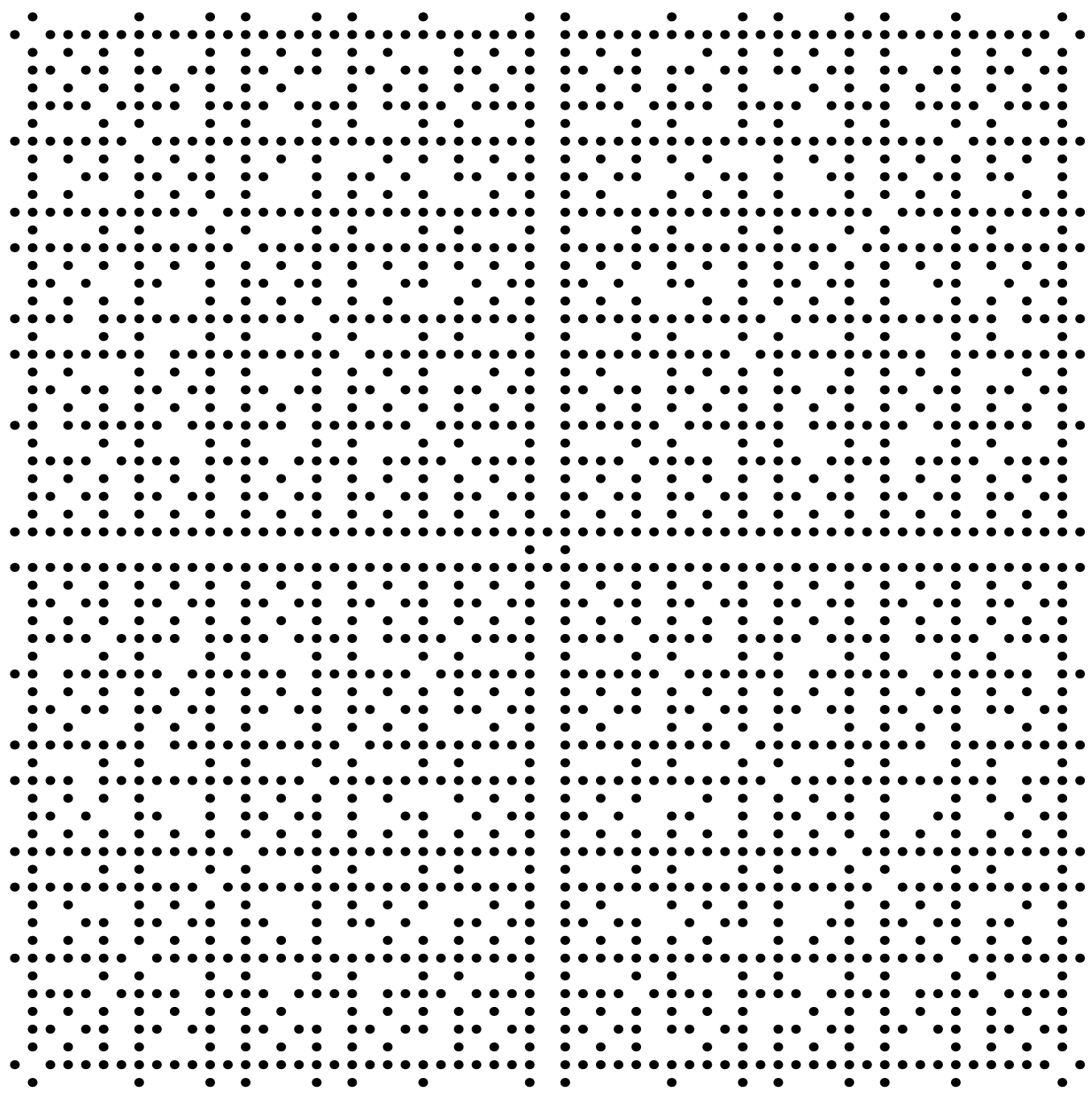

Figure 1: Some visible points of the 2D square lattice. 


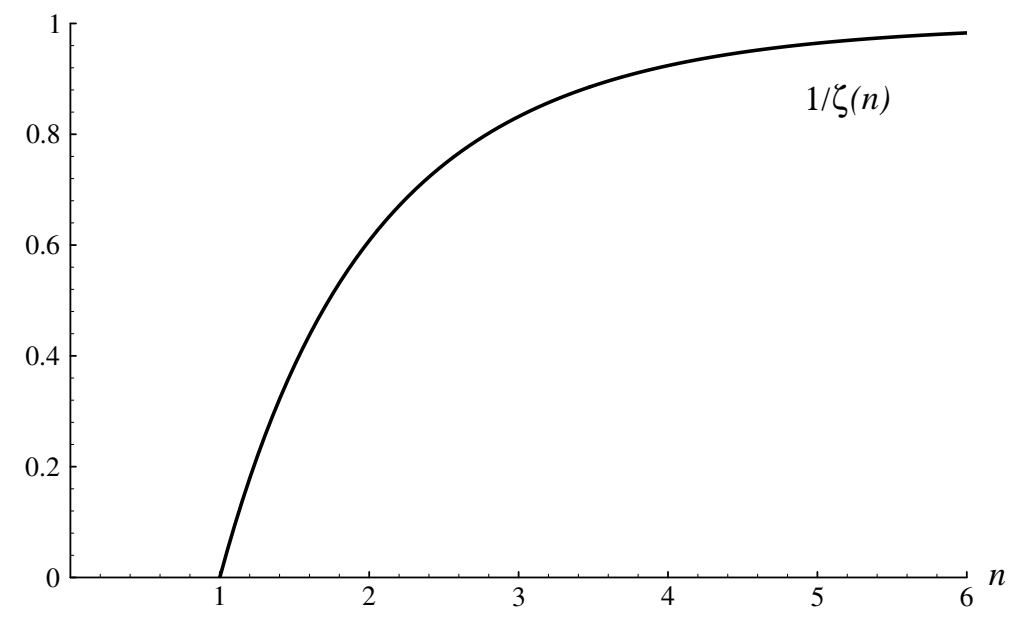

Figure 2: Relative frequency of visible points in a lattice as a function of the dimension. 


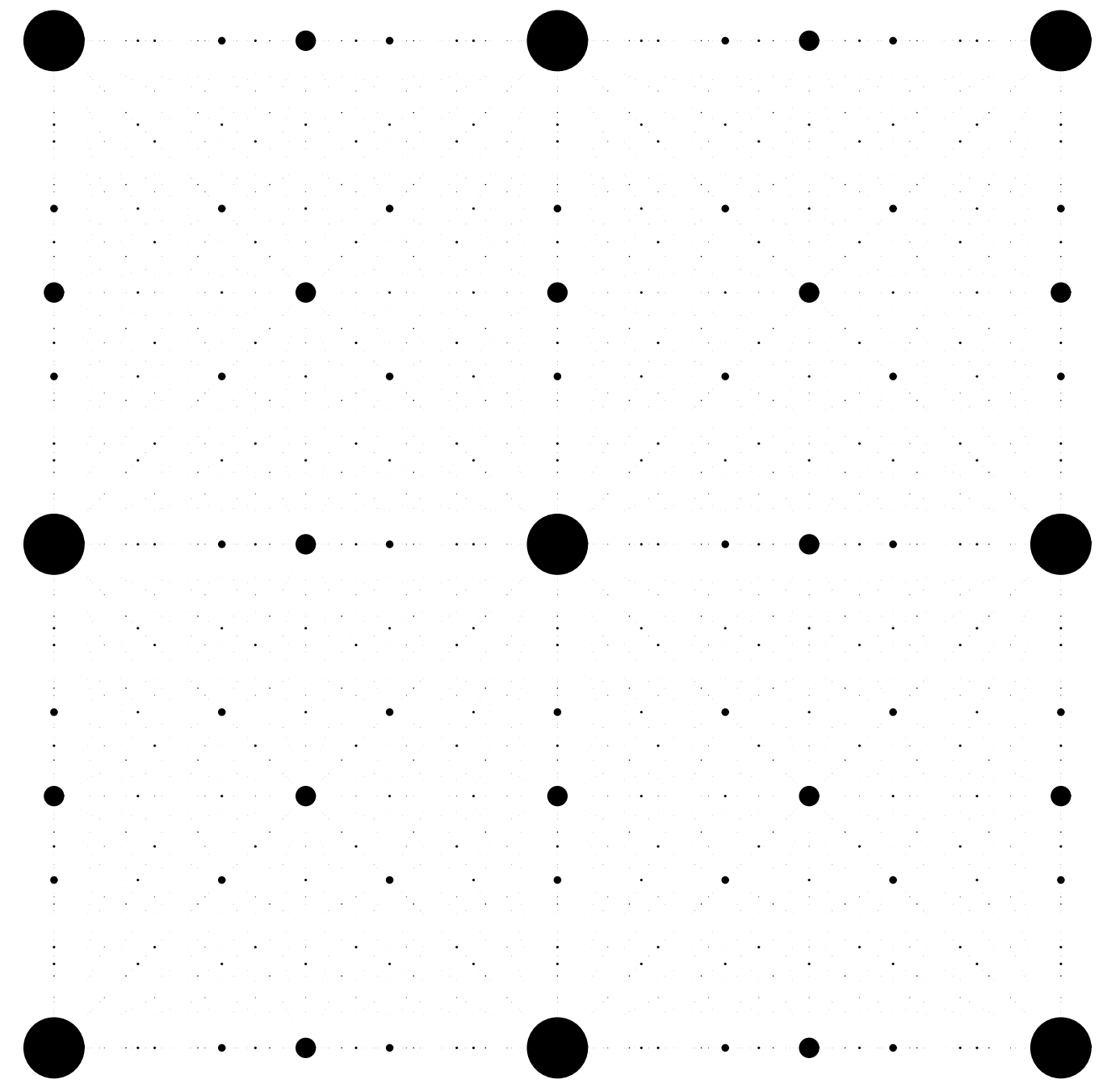

Figure 3: Bragg part of the Fourier transform of the square lattice according to (16). Here, the radius of the dots representing the Bragg peaks are proportional to the amplitude. 
Figure 4: Optical Fourier transform of some 9000 visible points of the square lattice $\mathbb{Z}^{2}$, see text for details. 
This figure "diffrac.jpg" is available in "jpg" format from: http://arxiv.org/ps/math-ph/9903046v1 